\title{
Fatores de risco para incontinência urinária em idosos institucionalizados
}

\author{
Risk factors for urinary incontinence in institutionalized elderly
}

Factores de riesgo para incontinencia urinaria en ancianos institucionalizados

Cíntia Lira Borges, ,* Bruna Karen Cavalcante Fernandes², Maria Lígia Silva Nunes Cavalcante², Rachel Gabriel Bastos Barbosa ${ }^{3}$, Arnaldo Aires Peixoto Junior ${ }^{4}$, Luciana Catunda Gomes de Menezes²

ORCID IDS

Borges CL (D) https://orcid.org/0000-0002-5204-0173

Fernandes BKC (D) https://orcid.org/0000-0003-2808-7526

Cavalcante MLSN (iD https://orcid.org/0000-0003-4830-9413

Barbosa RGB (D) https://orcid.org/0000-0003-4205-6910

Peixoto Junior AA (D) https://orcid.org/0000-0001-6225-934X

Menezes LCG (iD https://orcid.org/0000-0002-7828-9065

\section{COMO CITAR}

Borges CL, Fernandes BKC, Cavalcante MLSN, Barbosa RGB, Peixoto Junior AA, Menezes LCG. Fatores de risco para incontinência urinária em idosos institucionalizados. ESTIMA, Braz. J. Enterostomal Ther., 16: e0619. https://doi.org/10.30886/estima.v16.726 PT

\section{RESUMO}

Objetivo: Analisar os fatores de risco para incontinência urinária (IU) em idosos institucionalizados. Métodos: Estudo transversal, analítico, documental realizado por coleta em 203 prontuários de uma instituição de longa permanência no nordeste do Brasil. Foram utilizados um instrumento para caracterização sociodemográfica e de saúde e os valores do Mini Exame do Estado Mental (MEEM). Os dados foram analisados a partir da estatística descritiva, inferencial e por regressão logística múltipla. Resultados: A prevalência de IU foi de 42,36\% e maior em mulheres (66,28\%), mais velhos (média: 79,62 anos) e dependentes (89,53\%). Houve associação entre idade $(p=0,01)$, sexo $(p=0,024)$, estado civil $(p=0,007)$, religião $(p=0,015)$, com quem residia antes da institucionalização ( $p=0,033)$, grau de dependência ( $p<0,001)$, valores do MEEM $(p<0,001)$ e doenças cardiovasculares $(p<0,001)$ e neurológicas ( $p<0,001)$. Os fatores de risco para IU foram quantidade de doenças [odds ratio $(O R)=1,17$; intervalo de confiança de 95\% (IC95\%) = 1,02-1,34], consumo de cinco a nove medicamentos (OR = 4,07; IC95\% =1,14-14,52), ser solteiro (OR = 2,09; IC95\% =1,30-8,01), dependente $(\mathrm{OR}=3,27$; IC95\% = 1,33-8,04) e ter baixa pontuação no MEEM (OR = 0,88; IC95\% = 0,85-0,93). Conclusão: Os resultados revelam a importância de estudar IU nessa população para identificar precocemente os fatores de risco passíveis de prevenção.

DESCRITORES: Incontinência urinária; Idoso; Instituição de longa permanência para idosos; Fatores de risco; Prevalência; Estomaterapia.

1.Universidade Estadual do Ceará - Programa de Pós-Graduação em Saúde Coletiva - Fortaleza/CE - Brasil.

2.Universidade Estadual do Ceará - Programa Pós-Graduação em Cuidados Clínicos em Enfermagem e Saúde - Fortaleza/CE - Brasil.

3.Universidade Federal do Ceará - Departamento de Enfermagem - Fortaleza/CE - Brasil.

4.Centro Universitário Christus - Departamento de Medicina - Fortaleza/CE - Brasil.

*Autor correspondente: cintialiraborges@yahoo.com.br

Recebido: Fev. 26, 2019 | Aceito: Abr. 22, 2019 


\begin{abstract}
Objectives: To analyze the risk factors for urinary incontinence (UI) in institutionalized elderly. Methods: Cross-sectional, analytical, documentary study done by collection in 203 medical records of a long-stay institution in northeastern Brazil. An instrument for sociodemographic and health characterization and the values of the Mini Mental State Examination (MMSE) were used. The data were analyzed from the descriptive, inferential and multiple logistic regression. Results: The prevalence of UI was $42.36 \%$ and higher in women (66.28\%), older (mean: 79.62 years) and dependent (89.53\%). There was an association between age $(p=0.01)$, sex $(p=0.024)$, marital status $(p=0.007)$, religion $(p=0.015)$, with whom resided before institutionalization ( $p=0.033$ ), degree of dependency $(p<0.001)$, MMSE values $(p<0.001)$ and cardiovascular $(p<0.001)$ and neurological diseases $(p<0,001)$. The risk factors for $\mathrm{Ul}$ were number of diseases $(\mathrm{OR}=1.17 ; 95 \%$ $\mathrm{Cl}=1.02-1.34)$, consumption of five to nine medications $(\mathrm{OR}=4.07,95 \% \mathrm{Cl}=1.14-14.52)$, single $(\mathrm{OR}=2.09,95 \% \mathrm{Cl}=1.30-8.01)$, dependent $(\mathrm{OR}=3.27,95 \% \mathrm{Cl}=1.33-8.04)$ and had a low MMSE score $(\mathrm{OR}=0.88,95 \% \mathrm{Cl}=0.85-0.93)$. Conclusion: The results reveal the importance of studying $\mathrm{UI}$ in this population to identify early risk factors that can be prevented.
\end{abstract}

DESCRIPTORS: Urinary incontinence; Elderly; Homes for the Aged; Risk factors; Prevalence; Stomatherapy.

\title{
RESUMEN
}

Objetivo: Analizar los factores de riesgo para la incontinencia urinaria (IU) en ancianos institucionalizados. Métodos: Estudio transversal, analítico, documental realizado por colecta en 203 prontuarios de una institución de larga permanencia en el nordeste de Brasil. Se utilizó un instrumento para caracterización sociodemográfica y de salud y los valores del Mini Examen del Estado Mental (MEEM). Los datos fueron analizados a partir de la estadística descriptiva, inferencial y por regresión logística múltiple. Resultados: La prevalencia de IU fue de 42,36\% y mayor en mujeres (66,28\%), más viejos (promedio: 79,62 años) y dependientes (89,53\%). Hubo asociación entre edad $(p=0,01)$, género $(p=0,024)$, estado civil $(p=0,007)$, religión $(p=0,015)$, con quienes residía antes de la institucionalización ( $p=0,033)$, grado de dependencia $(p<0,001)$, valores del MEEM $(p<0,001)$ y enfermedades cardiovasculares $(p<0,001)$ y neurológicas $(p<0,001)$. Los factores de riesgo para IU fueron la cantidad de enfermedades [odds ratio (OR) = 1,17; el intervalo de confianza del 95\% $(I C 95 \%)=1,02-1,34]$, el consumo de cinco a nueve medicamentos $(O R=4,07, I C 95 \%=1,14-14,52)$, ser soltero $(\mathrm{OR}=2,09 ; \mathrm{IC} 95 \%=1,30-8,01)$, dependiente $(\mathrm{OR}=3,27 ; \mathrm{IC} 95 \%=1,33-8,04)$ y tener baja puntuación en el MEEM $(\mathrm{OR}=0,88$; IC95\% = 0,85-0,93). Conclusión: Los resultados revelan la importancia de estudiar IU en esa población para identificar precozmente los factores de riesgo pasibles de prevención.

DESCRIPTORES: Incontinencia urinaria; Ancianos; Hogares para ancianos; Factores de riesgo; Prevalencia; Estomaterapia.

\section{INTRODUÇÃO}

A incontinência urinária (IU) é problema comum entre idosos institucionalizados, com consequências relevantes para a qualidade de vida (QV) e custos em saúde 1 . Pesquisas indicam que a IU pode ser o motivo de admissão em instituições de longa permanência para idosos ${ }^{2}$ ou pode ser facilmente desenvolvida após a institucionalização.

A prevalência da IU nesses locais pode variar de $35,9^{3}$ a $72 \%{ }^{4}$, valor consideravelmente maior quando comparado a idosos da comunidade, na qual a prevalência é de $3,5^{5}$ a $10,3 \%{ }^{6}$. Esse problema representa custos elevados para sociedade, família e idoso. Nos Estados Unidos, estima-se que, em 2007, foram gastos cerca de US\$ 65,9 bilhões, em 2015, US\$76,2 bilhões, e há projeções para, 2020, serem gastos, aproximadamente, US $\$ 82,6$ bilhões pelo sistema de saúde e pelos pacientes para compra de itens pessoais para cuidados de rotina com a IU7.
As causas de IU em idosos são diversas e nas mulheres estão mais associadas à idade, ao índice de massa corporal, à paridade, ao tabagismo, à histerectomia ${ }^{8}$, à hipertensão, ao diabetes e à menopausa ${ }^{9}$. Em homens, pode surgir devido à idade ${ }^{9}$, à hiperplasia prostática benigna ${ }^{10}$ ou no pós-cirúrgico de cirurgias, como resseção transuretral, prostatectomia aberta e radical retropúbica ${ }^{11}$.

Por definição, IU significa perda involuntária de urina ${ }^{12}$. Existem dois tipos de IU: a transitória e a persistente. A primeira é reversível, proveniente de condições externas que podem ser tratadas ${ }^{13}$. A segunda ocorre mesmo após os problemas transitórios serem resolvidos e divide-se em quatro grupos: de urgência (perda abrupta de urina após forte desejo para urinar); de esforço (perda involuntária associada ao esforço físico); de transbordamento (quando ocorrem perda e fuga de urina após exceder a capacidade total de armazenamento pela bexiga); e funcional (relaciona-se à incapacidade ou falta de vontade de ir ao banheiro) ${ }^{13}$. 
A IU gera consequências desastrosas para a vida do indivíduo, sobretudo o idoso que, muitas vezes, acredita que a IU é parte do envelhecimento natural e menospreza o problema. Porém, essa é uma condição crônica e debilitante que acarreta prejuízos físicos, sociais e emocionais. A IU é importante marcador de mortalidade e está relacionada a síndromes geriátricas e à fragilidade, podendo ser um determinante real de sobrevida na população institucionalizada ${ }^{1}$.

É importante identificar os fatores de risco para IU para que estratégias de prevenção e tratamentos eficazes sejam implantados precocemente, no intuito de evitar ou controlar a $\mathrm{IU}^{3}$. É recomendável realizar a avaliação funcional e de saúde do idoso, compreendendo mobilidade, transferência, habilidade para ir ao banheiro, perfil de doenças e medicamentos e causas da IU, a fim de planejar terapias comportamentais, como a miç̧ão programada ${ }^{14}$. Essas medidas combinadas a atividades físicas e exercícios específicos podem melhorar o status funcional e a condição de IU entre idosos ${ }^{3}$. Dessa forma, a partir da avaliação ampla da equipe multiprofissional, é possível detectar o tipo de $\mathrm{IU}$ e individualizar o plano de cuidados de acordo com as necessidades de cada idoso.

Ademais, a equipe de enfermagem tem importante papel no cuidado ao idoso incontinente. Existem inúmeras intervenções de enfermagem que podem ser determinantes para a qualidade dos cuidados em instituições, como a presença de enfermeiros com capacitação para o trabalho da continência, a disponibilização de folhetos informativos sobre IU aos residentes e cuidadores, o treinamento de exercícios para bexiga e relaxamento e a viabilização de protocolos de gestão organizados e documentados para garantia de produtos para IU - fraldas descartáveis, roupas confortáveis e almofadas descartáveis ou laváveis ${ }^{15}$. É preciso que o enfermeiro abandone a ideia de que a IU sempre é consequência normal do envelhecimento e acredite ser necessário preveni-la ativamente na prática de enfermagem ${ }^{15}$.

O objetivo desse estudo é analisar os fatores de risco para IU em idosos institucionalizados.

\section{MÉTODOS}

Estudo do tipo transversal, quantitativo, documental, retrospectivo, com utilização de 203 prontuários de idosos residentes em uma instituição de longa permanência sem fins lucrativos no nordeste do Brasil. O local atende idosos de todas as classes sociais, predominantemente aqueles em situação de vulnerabilidade social, sendo a admissão por vontade própria, violência ou encaminhamento de órgãos e instituições públicas. A coleta ocorreu entre os dias 1 e 30 de setembro de 2016.

Os dados foram coletados a partir de um instrumento para caracterização sociodemográfica e de saúde que continha perguntas sobre idade, sexo, estado civil, escolaridade, religião, motivo e tempo de institucionalização, com quem residia antes da institucionalização, visita, doenças, medicamentos e grau de dependência. Além disso, foram examinados os resultados da última avaliação do Mini Exame do Estado Mental (MEEM) ${ }^{16}$, o qual é realizado trimestralmente, nessa instituição, pelos profissionais de saúde.

A IU, variável desfecho, foi definida conforme as especificações dos diagnósticos e a lista de problemas médicos dos prontuários. Apesar de existir informações acerca da especificação da IU, por exemplo, IU de urgência e IU mista, nesse estudo, para análise estatística, a variável IU foi dicotômica, dividindo-se em presença ou ausência, independentemente do tipo.

Sobre a variável com quem residia antes da institucionalização, a categoria "outros" se referiu a amigos, parentes, abrigos ou instituições de saúde. Da mesma forma, o motivo de institucionalização na categoria outros evidenciou idosos que sofreram violência, eram moradores de rua, foram transferidos de outros abrigos ou encaminhados pelo Ministério Público. O tempo de institucionalização foi medido em meses e os medicamentos foram distribuídos em três categorias, as quais foram divididas conforme a definição de polifarmácia (de cinco a nove medicamentos) e polifarmácia excessiva (mais de 10 medicamentos $)^{17}$.

O grau de dependência foi caracterizado por níveis definidos em: I. idoso independente; II. idoso com dependência em até três atividades básicas de vida diária, sem ou com alteração cognitiva controlada; III. idosos com dependência em todas as atividades de autocuidado e/ou com comprometimento cognitivo ${ }^{18}$. Essa classificação é atualizada mensalmente, pois é item obrigatório do regulamento técnico da Agência Nacional de Vigilância Sanitária (Anvisa) para funcionamento de instituições de longa permanência para idosos, bem como, constantemente, o local é submetido à fiscalização, avaliação e controle social e necessita dessa avaliação para organização das ações e dos cuidados aos residentes. 
O MEEM é um teste que avalia a função cognitiva de forma rápida, prática e simples ${ }^{16}$. É utilizado em diferentes contextos da prática clínica para rastreios cognitivos, identificando as principais funções que merecem ser investigadas ${ }^{19}$. Não foi utilizado ponto de corte do MEEM nesse estudo, uma vez que a variável foi numérica, e não categórica.

Para elaboração da tabela e categorização dos tipos de doenças, fez-se uma lista com as doenças de todos os idosos, as quais foram distribuídas conforme as classes: cardiovascular, neurológica, psiquiátrica, osteomuscular, endócrina e renal.

A análise foi realizada por meio do programa Stata versão 11.0. Os dados foram expressos com médias, desvio padrão, mínimo e máximo para variáveis contínuas, e frequências e porcentagens para variáveis categóricas. Adotou-se o teste de Shapiro-Wilk, com nível de significância $<0,05$, concluindo-se que todas as variáveis contínuas (idade, tempo de institucionalização e número de doenças) não se encontravam no padrão de normalidade, rejeitando-se a hipótese nula. Por isso, para associação bivariada entre essas e a IU, aplicou-se o teste não paramétrico de Mann-Whitney. Para associação da IU e das variáveis categóricas, empregou-se o teste qui-quadrado de Pearson.

$\mathrm{Na}$ análise final dos fatores associados, utilizou-se o modelo de regressão logística. Para a modelagem, estado civil (solteiro e outros), religião (católica e outros), motivo de institucionalização (sozinho e outros) e grau de dependência (independente/grau I e dependente/graus II e III) foram transformadas em variáveis dicotômicas.

Primeiramente, construiu-se um modelo reduzido inicial com categorias basais, a partir da regressão logística simples. Desse modelo, incluíram-se as variáveis que apresentaram nível descritivo de até 20\% ( $p<0,20)$. Nesse momento, excluíram-se as variáveis tempo de institucionalização e visita. A próxima etapa foi a criação do modelo multivariado ajustado para grau de dependência, permanecendo aquelas com $\mathrm{p} \leq 0,2$, sendo excluído sexo $(\mathrm{p}=0,275)$, motivo de institucionalização $(\mathrm{p}=0,867)$ e com quem residia antes da institucionalização $(p=0,282)$. Destaca-se que a variável número de doenças, nessa etapa, apresentou $\mathrm{p}=0,246$, mas foi mantida.

O ajuste global de cada modelo foi verificado pelo teste da razão de verossimilhança, sendo o modelo de regressão logística múltipla inicial e final considerados iguais $(p=0,43)$.
Por essa razão, adotou-se o que continha menos variáveis. No modelo final, permaneceram aquelas variáveis com $\mathrm{p}<0,05$, além da variável estado civil que, apesar de ter $\mathrm{p}=0,066$, foi conservada. A medida de associação foi representada pelo odds ratio (OR), com intervalo de confiança de 95\% (IC95\%).

Foram atendidas as recomendações éticas sobre pesquisas com seres humanos, conforme a Resolução 466/2012 do Ministério da Saúde ${ }^{20}$. A pesquisa foi aprovada pelo Comitê de Ética da Universidade Estadual do Ceará (CAAE: 12390513.8.0000.5534; parecer: 1.532.812).

\section{RESULTADOS}

Prevaleceram os idosos do sexo feminino $(57,14 \%)$ com idade média de 77,59 anos $( \pm 8,99)$. A prevalência de IU foi de $42,36 \%$ e maior em mulheres idosas $(66,28 \%)$, nos mais velhos (média: 79,62 anos) e dependentes (89,53\%). Na Tabela 1, observa-se associação estatisticamente significativa entre idade $(p=0,01)$, sexo $(p=0,024)$, estado civil $(p=0,007)$, religião $(p=0,015)$ e com quem residia antes $\mathrm{da}$ institucionalização $(\mathrm{p}=0,033)$.

$\mathrm{Na}$ Tabela 2, houve relação entre grau de dependência ( $p<0,001)$ e valores do MEEM ( $<<0,001)$.

A Tabela 3 mostra que houve relação significativa entre IU e doenças cardiovasculares $(\mathrm{p}<0,001)$ e neurológicas $(\mathrm{p}<0,001)$.

$\mathrm{Na}$ análise univariada, no modelo simples, demonstrada na Tabela 4, somente duas variáveis, visita e tempo de institucionalização, não estiveram relacionadas com o desfecho de IU. Já no modelo multivariado ajustado para grau de dependência, permaneceram todas as variáveis, exceto sexo, motivo de institucionalização, tempo de institucionalização e com quem residia antes da institucionalização.

No modelo final de regressão logística, na Tabela 5, conservaram-se as variáveis estado civil $(\mathrm{OR}=2,09$; IC95\% = 1,30-8,01), doenças (OR = 1,17; IC95\% = 1,02-1,34), medicamentos [de um a quatro $(\mathrm{OR}=3,90 ; \mathrm{IC} 95 \%=0,98$ $15,46)$ e de cinco a nove $(\mathrm{OR}=4,07 ; \mathrm{IC} 95 \%=1,14-14,52)]$, grau de dependência $(\mathrm{OR}=3,27$; IC $95 \%=1,33-8,04)$ e $\operatorname{MEEM}(\mathrm{OR}=0,88 ; \mathrm{IC} 95 \%=0,85-0,93)$. A maior quantidade de doenças, polifarmácia, ser dependente e solteiro são fatores de risco importantes para o acometimento da IU. Os valores do MEEM foram inversamente proporcionais à presença de $\mathrm{IU}$, de forma que quanto menos pontos no teste cognitivo, maiores as chances de ter IU. 
Tabela 1. Relação entre o perfil social e de institucionalização dos idosos institucionalizados e a incontinência urinária (IU), Fortaleza, estado do Ceará, Brasil, 2016.

\begin{tabular}{|c|c|c|c|}
\hline \multirow{2}{*}{ Variáveis independentes } & \multicolumn{2}{|c|}{ Incontinência urinária } & \multirow{2}{*}{ p-valor } \\
\hline & Ausência f (\%) & Presença $\mathrm{f}(\%)$ & \\
\hline Idade (mínimo: 60; máximo: 103 anos) & $76,09( \pm 7,89)^{*}$ & $79,62( \pm 9,99)^{*}$ & $0,01^{+}$ \\
\hline Sexo & & & $0,024^{\ddagger}$ \\
\hline Masculino & $58(49,57)$ & $29(33,72)$ & \\
\hline Feminino & $59(50,43)$ & $57(66,28)$ & \\
\hline Estado civil & & & $0,007^{\ddagger}$ \\
\hline Solteiro & $49(41,88)$ & $52(60,47)$ & \\
\hline Separado/divorciado & $39(33,33)$ & $11(12,79)$ & \\
\hline Viúvo & $26(22,22)$ & $20(23,26)$ & \\
\hline Casado & $03(2,56)$ & $03(3,49)$ & \\
\hline Religião & & & $0,015^{\ddagger}$ \\
\hline Católica & $93(79,49)$ & $79(91,86)$ & \\
\hline Outras & $24(20,51)$ & $07(8,14)$ & \\
\hline Recebe visita & & & $0,848^{\ddagger}$ \\
\hline Sim & $75(64.10)$ & $54(62.79)$ & \\
\hline Não & $42(35.90)$ & $32(37.21)$ & \\
\hline Com quem residia antes da institucionalização & & & $0,033^{\ddagger}$ \\
\hline Sozinho & $57(48,72)$ & $31(36,05)$ & \\
\hline Filhos & $13(11,11)$ & $22(25,58)$ & \\
\hline Cônjuges & $11(9,40)$ & $05(5,81)$ & \\
\hline Outros & $36(30,77)$ & $28(32,56)$ & \\
\hline Motivo de institucionalização & & & $0,062^{\ddagger}$ \\
\hline Vontade própria & $43(36,75)$ & $21(24,42)$ & \\
\hline Outros motivos & $74(63,25)$ & $65(75,58)$ & \\
\hline Tempo de institucionalização (mínimo: 6; máximo: 648 meses) & $91,94( \pm 88,50)^{*}$ & $105,73( \pm 120,83)^{\star}$ & $0,685^{\dagger}$ \\
\hline
\end{tabular}

*Média; †Teste de Mann-Whitney; ‘Teste qui-quadrado.

Tabela 2. Relação entre o perfil de saúde dos idosos institucionalizados e a incontinência urinária (IU), Fortaleza, estado do Ceará, Brasil, 2016

\begin{tabular}{|c|c|c|c|}
\hline \multirow{2}{*}{ Variáveis independentes } & \multicolumn{2}{|c|}{ Incontinência urinária } & \multirow{2}{*}{ p-valor } \\
\hline & Ausência f (\%) & Presença $f(\%)$ & \\
\hline Número de doenças (mínimo: 2; máximo: 17) & $5,76( \pm 2,64)^{\star}$ & $6,52( \pm 3,43)^{*}$ & $0,219^{+}$ \\
\hline Medicamentos & & & $0,073^{\ddagger}$ \\
\hline 1 a 4 & $39(33,33)$ & $34(39,53)$ & \\
\hline 5 a 9 & $57(48,72)$ & $46(53,49)$ & \\
\hline 10 a 16 & $21(17,95)$ & $6(6,98)$ & \\
\hline Grau de dependência & & & $<0,001^{\ddagger}$ \\
\hline 1 & $64(54,7)$ & $09(10,47)$ & \\
\hline 2 & $53(45,3)$ & $12(13,95)$ & \\
\hline 3 & - & $65(75,58)$ & \\
\hline Mini Exame do Estado Mental (mínimo: 0; máximo: 30) & $17,70( \pm 8,20)^{*}$ & $6,96( \pm 7,90)^{\star}$ & $<0,001^{\ddagger}$ \\
\hline
\end{tabular}

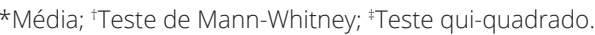


Tabela 3. Relação entre classes de doenças e incontinência urinária (IU) em idosos institucionalizados, Fortaleza, estado do Ceará, Brasil, 2016.

\begin{tabular}{|c|c|c|c|}
\hline \multirow{2}{*}{ Doenças } & \multicolumn{2}{|c|}{ Incontinência urinária } & \multirow{2}{*}{ p-valor } \\
\hline & Ausência f (\%) & Presença $f(\%)$ & \\
\hline Cardiovasculares & $86(57,2)$ & $42(32,8)$ & $<0,001^{*}$ \\
\hline Neurológicas & $46(42,2)$ & $63(57,8)$ & $<0,001^{*}$ \\
\hline Psiquiátricas & $57(55,9)$ & $45(44,1)$ & $0,611 *$ \\
\hline Osteomusculares & $47(54)$ & $40(46)$ & $0,367^{\star}$ \\
\hline Endócrinas & $46(66,7)$ & $23(33,3)$ & $0,62^{*}$ \\
\hline Renais & $22(73,3)$ & $08(26,7)$ & $0,59 *$ \\
\hline
\end{tabular}

*Teste qui-quadrado.

Tabela 4. Fatores associados à presença de incontinência urinária (IU) em idosos institucionalizados no modelo de regressão logística simples para cada variável independente e modelo multivariado inicial ajustado para grau de dependência, Fortaleza, estado do Ceará, Brasil, 2016.

\begin{tabular}{|c|c|c|c|c|c|c|}
\hline \multirow{2}{*}{ Variáveis (categoria basal) } & \multicolumn{3}{|c|}{ Modelo simples } & \multicolumn{3}{|c|}{ Modelo multivariado ajustado } \\
\hline & ORb & IC & p-valor & ORa & IC & p-valor \\
\hline Idade & 1,04 & $1,01-1,08$ & 0,006 & 1,03 & $0,99-1,06$ & 0,076 \\
\hline Sexo (masculino) & 1,93 & $1.08-3,43$ & 0,025 & 1,04 & $0,75-2,73$ & 0,275 \\
\hline Estado civil (outros) & 2,12 & $1,20-3,74$ & 0,009 & 1,73 & $0,92-3,26$ & 0,088 \\
\hline Religião (outras) & 2,91 & $1,19-7,11$ & 0,019 & 3,12 & $1,18-8,21$ & 0,021 \\
\hline Recebe visita (não recebe) & 0,94 & $0,53-1,68$ & 0,848 & - & - & - \\
\hline Com quem residia antes da institucionalização (sozinho) & 1,68 & $0,95-2,98$ & 0,073 & 1,41 & $0,74-2,68$ & 0,283 \\
\hline Motivo de institucionalização (vontade própria) & 1,79 & $0,96-3,34$ & 0,063 & 0,93 & $0,45-1,96$ & 0,867 \\
\hline Tempo de institucionalização & 1,00 & $0,99-1,00$ & 0,350 & - & - & - \\
\hline Número de doenças & 1,08 & $0,99-1,19$ & 0,078 & 1,06 & $0,95-1,17$ & 0,246 \\
\hline \multicolumn{7}{|l|}{ Medicamentos (10 a 18) } \\
\hline 1 a 4 & 3,05 & $1,10-8,43$ & 0,032 & 3,24 & $1,08-9,66$ & 0,035 \\
\hline 5 a 9 & 2,82 & $1,05-7,57$ & 0,039 & 3,76 & $1,29-10,97$ & 0,015 \\
\hline Grau de dependência (I) & 10,33 & $4,73-22,54$ & 0,000 & 10,33 & $4,73-22,54$ & $<0,001$ \\
\hline Mini Exame do Estato Mensal (MEEM) & 0,86 & $0,83-0,90$ & 0,000 & 0,89 & $0,85-0,93$ & $<0,001$ \\
\hline
\end{tabular}

IC = intervalo de confiança; ORa = odds ratio ajustada; ORb = odds ratio bruta.

Tabela 5. Fatores associados à presença de incontinência urinária (IU) em idosos institucionalizados no modelo de regressão logística múltipla inicial e final, Fortaleza, estado do Ceará, Brasil, 2016.

\begin{tabular}{|c|c|c|c|c|c|c|}
\hline \multirow{2}{*}{ Variáveis (categoria basal) } & \multicolumn{3}{|c|}{ Modelo multivariado inicial } & \multicolumn{3}{|c|}{ Modelo multivariado final } \\
\hline & $\mathrm{ORb}$ & IC & p-valor & ORa & IC & p-valor \\
\hline Idade & 1,00 & $0,96-1,04$ & 0,777 & - & - & - \\
\hline Estado civil (outros) & 1,99 & $0,95-4,17$ & 0,066 & 2,09 & $1,00-4,33$ & 0,047 \\
\hline Religião (outras) & 1,91 & $0,66-5,54$ & 0,231 & - & - & - \\
\hline Número de doenças & 1,16 & $1,01-1,33$ & 0,034 & 1,17 & $1,02-1,34$ & 0,023 \\
\hline \multicolumn{7}{|l|}{ Medicamentos (10 a 18) } \\
\hline 1 a 4 & 3,78 & $0,94-15,20$ & 0,061 & 3,90 & $0,98-15,46$ & 0,052 \\
\hline 5 a 9 & 3,73 & $1,03-13,49$ & 0,044 & 4,07 & $1,14-14,52$ & 0,030 \\
\hline Grau de dependência (I) & 3,19 & $1,28-7,97$ & 0,013 & 3,22 & $1,30-8,01$ & 0,012 \\
\hline Mini Exame do Estato Mensal (MEEM) & 0,89 & $0,84-0,93$ & 0,000 & 0,88 & $0,84-0,93$ & $<0,001$ \\
\hline
\end{tabular}

IC = intervalo de confiança; MEEM = Mini Exame do Estado Mental; ORa = odds ratio ajustada; ORb = odds ratio bruta. 


\section{DISCUSSÃO}

A prevalência de IU não se mostrou discordante de outros estudos nacionais e internacionais ${ }^{1,3,4,21,22}$. Apesar de a literatura apresentar diferenças significativas acerca da IU entre gêneros na comunidade, tendo a mulher frequência relativa dobrada de adquirir IU em comparação ao homem ${ }^{23,24}$, em instituições, ao contrário, é semelhante a variação da prevalência entre os sexos. Em idosos, varia de $33,9^{4}$ a $60,3 \%{ }^{21}$, e, em idosas, de $31,5^{4}, 47,5^{22}$ e 59,2\% ${ }^{21}$. Acredita-se que essa diferença da comunidade para a instituição de longa permanência justifica-se porque os idosos estão mais propensos a um maior número de comorbidades, polifarmácia e restrições físicas que interferem na mobilidade, nutrição e uso do banheiro ${ }^{14}$. Nessa pesquisa, predominaram as mulheres $(p=0,024)$ e os mais velhos $(p=0,01)$.

Embora não haja grande diferença da frequência de IU entre idosos do sexo masculino e feminino, destaca-se que o risco de morte associado à IU de idosos institucionalizados, usuários da polifarmácia e com algum grau de dependência é maior do que entre idosas na mesma condição ${ }^{1}$. Ressalta-se que o tempo de permanência também é preditor para a gravidade da IU ${ }^{4}$.

A IU é relatada por muitos como condição incapacitante e constrangedora. $\mathrm{O}$ fato de se sentir mal ou com vergonha por ter a doença e solicitar ajuda pode afetar negativamente os comportamentos de busca de cuidados ${ }^{25}$. Porém, ao conviver muito tempo com o problema, o estigma e a rejeição social podem deixar de ser uma barreira substancial e transformar-se em fatores-chave para a procura de cuidados em saúde ${ }^{25}$. Em instituições de longa permanência, até mesmo pela fragilidade emocional e social que envolve esses ambientes, a política de prevenção deve ser redobrada. É necessário que os profissionais não esperem, somente, que os idosos relatem o problema espontaneamente, mas estimulem o autocuidado, estejam disponíveis para as demandas e realizem uma busca ativa dos sinais e sintomas comuns que abrangem a IU, de forma sutil e dialogando com os idosos.

Nos próximos anos, irá aumentar a quantidade de idosos moradores de instituições de longa permanência e que vivem sozinhos $^{26}$. Viver sozinho exerce impacto direto no estado de saúde dos idosos, os quais podem ter mais doenças e deficiências ${ }^{27}$. Para muitos idosos do sexo masculino, morar sozinho resulta em falta de apoio social, distúrbios cognitivos, depressão, doenças crônicas e quedas ${ }^{27}$. Para as idosas, morar sozinha e ser solteira pode representar ocorrência de quedas, múltiplas doenças, depressão e dor crônica ${ }^{27}$. Ser solteiro, também, é determinante para restringir o idoso em sua casa ${ }^{5}$. Nesse estudo, ser solteiro correspondeu 2,09 mais chances de ter IU, comparado com viúvos, separados, divorciados e casados. E morar sozinho antes da institucionalização ( $p=0,033)$ esteve relacionado com IU.

Vários estudos referem que a presença de múltiplas comorbidades, especialmente as cardiovasculares e neurológicas, é fator de risco para IU. Um estudo brasileiro encontrou que o acidente vascular encefálico $(\mathrm{OR}=1,62)$ e a doença de Alzheimer $(\mathrm{OR}=1,38)$ foram as únicas positivamente associadas à $\mathrm{IU}^{21}$, e que idosos residentes de instituições com grave declínio cognitivo tinham risco aumentado $(\mathrm{OR}=1,92)$ para desenvolvimento de $\mathrm{IU}^{21}$. $\mathrm{Na}$ Espanha, uma coorte verificou que 80,6\% dos idosos residentes incontinentes tinham demência $(\mathrm{p}<0,001)^{1} \mathrm{e}$ que os idosos com insuficiência cardíaca $(\mathrm{OR}=1,88)$ e arritmias $(\mathrm{OR}=1,51)$ tinham maior risco de mortalidade relacionada à $\mathrm{IU}^{1}$. O presente estudo detectou associação significante entre IU e a classe de doenças cardiovasculares e neurológicas e risco de 1,17 para desenvolver IU naqueles que tenham mais doenças.

A polifarmácia, definida como uso de múltiplas drogas ou uso de mais de um medicamento desnecessário,é preocupação crescente na população geriátrica ${ }^{28}$. Evidências atuais indicam que o uso é maior em instituições de longa permanência e que quase $50 \%$ dos idosos tomam um ou mais medicamentos dispensáveis ${ }^{28}$. Muitos desses podem causar aumento da frequência urinária ${ }^{29}$.

No modelo de regressão múltipla, a polifarmácia $(\mathrm{OR}=4,07)$ foi fator de risco para IU. É indispensável a avaliação dos profissionais de saúde no propósito de identificar se a IU é manifestada como efeito colateral potencial dos medicamentos prescritos. Isso porque contribui para a redução da prescribing cascade, que ocorre quando um novo medicamento é adicionado para tratar essencialmente um fator colateral de uma terapêutica já implementada ${ }^{30}$. Esse achado chama a atenção para intensificar a supervisão de medicamentos que possam corroborar o problema.

Em instituições de longa permanência, a dependência é um determinante direto da IU. No presente estudo, observou-se risco triplo entre idosos dependentes para desenvolvimento da IU. Uma pesquisa confirma risco de 3,6 e 5,34 de idosos dependentes adquirirem IU e indica 
que $90,47 \%$ dos idosos dependentes tinham IU ${ }^{21}$. O declínio funcional e da mobilidade pode justificar o aparecimento de IU, como resultado de banheiros inacessíveis, incapacidade de retirar roupas a tempo, descoordenação do músculo pélvico e outras causas multifatoriais ${ }^{3}$. A IU também pode limitar a mobilidade dos pacientes, favorecendo um estilo de vida menos ativo, com comprometimento das relações pessoais, gerando, em longo prazo, depressão, incapacidade e morte ${ }^{1}$.

Ressalta-se que os músculos do assoalho pélvico atuam como parte da unidade abdominopélvica, por isso exercícios que integrem força e coordenação do tronco ou da musculatura abdominal proporcionam um funcionamento eficiente do músculo do assoalho pélvico, impedindo episódios de $\mathrm{UI}^{3}$; além disso, contribuem para minimizar o avanço dos níveis de dependência e melhorar a mobilidade.

A IU pode preceder ou acompanhar o surgimento de condições neurológicas, incluindo doença de Alzheimer, demência multi-infarto, acidente vascular cerebral e doença de Parkinson ${ }^{31}$. A função do centro de micção no tronco encefálico é controlada pelo lobo frontal e, no contexto de doenças neurodegenerativas ou acidente vascular encefálico, ocorre disfunção do córtex frontal, dando origem à IU ${ }^{32}$.

Outras evidências concordam com esse estudo quando referem que a prevalência de IU aumenta com a diminuição dos escores do $\mathrm{MEEM}^{31}$. Já são bem definidos na literatura o efeito do desempenho cognitivo sobre a IU e o aspecto bidirecional entre essas duas condições ${ }^{32}$. Além disso, idosos mais velhos e dependentes moradores de instituições apresentam condição cognitiva menor em comparação aos da comunidade ${ }^{33}$.

Revela-se que a assistência de enfermagem à IU, em todos os casos, realizada por profissionais treinados, afeta positivamente a gravidade da IU e o impacto da $\mathrm{QV}^{34}$. $\mathrm{O}$ enfermeiro deve conhecer os aspectos associados à IU, bem como intervir precocemente, compreendendo que os resultados em idosos são lentos, mas devem ser sustentados para proporcionar independência, autonomia e melhores condições de vida.

A associação entre IU e algumas variáveis estudadas pode ser uma via de mão dupla. A partir dessa pesquisa, foram estudados os fatores de risco para IU. Entretanto, estudos adicionais longitudinais devem ser realizados para estabelecer essa relação, atentando que fatores, muitas vezes, considerados inócuos podem desencadear diversos desfechos clínicos negativos em instituições de longa permanência.
As limitações desse estudo foram: 1) estudo transversal o qual não permite o estabelecimento de uma relação causa-efeito entre as variáveis e, portanto, os dados devem ser analisados com cuidado; 2) a instituição pesquisada é uma organização não governamental, carente de recursos e, constantemente, recebe idosos com estado de saúde geral precário, por isso, não é possível estabelecer se os idosos incontinentes já foram admitidos com o problema ou se desenvolveram na instituição; 3) o MEEM depende da variável escolaridade coletada, mas não foi avaliada nesse estudo; 4) os subtipos da IU não foram explorados, apesar de definidos nos prontuários; 5) o conjunto de variáveis para ajuste poderia ter sido um pouco mais abrangente, pois ainda é possível haver fatores confundidores residuais na análise.

\section{CONCLUSÃO}

Foi constatada relação entre as variáveis independentes ser solteiro, ter múltiplas comorbidades, ser usuário da polifarmácia, dependente e apresentar declínio cognitivo, fatores determinantes predominantes para IU em idosos institucionalizados.

Os resultados revelam a importância de estudar IU em idosos institucionalizados, tendo em vista a quantidade escassa de pesquisas no Brasil e a reduzida abordagem clínica pelos profissionais de saúde. Além disso, os fatores de risco detectados são evitáveis, passíveis de prevenção, desde que identificados precocemente.

Prevenir ou melhorar a IU por meio de cuidados e atenção minimiza os índices de mortalidade em idosos institucionalizados e contribui para melhor QV e independência funcional.

\section{CONTRIBUIÇÃO DOS AUTORES}

Conceitualização, Borges CL; Metodologia, Borges CL e Fernandes BKC; Investigação, Borges CL e Fernandes BKC; Redação - Primeira versão, Borges CL e Menezes LCG; Redação - Revisão \& Edição, Borges CL, Menezes LCG, Cavalcante MLSN, Barbosa RGB e Peixoto Junior AA.; Aquisição de Financiamento, Borges CL; Recursos, Borges CL; Supervisão, Borges CL. 


\section{REFERÊNCIAS}

1. Damián J, Pastor-Barriuso R, García López FJ, Pedro-Cuesta J. Urinary incontinence and mortality among older adults residing in care homes. J Adv Nurs. 2017;73(3):688-99. https://doi.org/10.1111/jan.13170

2. Maxwell CJ, Soo A, Hogan DB, Wodchis WP, Gilbart E, Amuah J, et al. Predictors of nursing home placement from assisted living settings in Canada. Can J Aging. 2013;32(4):333-48. https://doi.org/10.1017/S0714980813000469

3. Chiu AF, Huang MH, Hsu MH, Liu JL, Chiu JF. Association of urinary incontinence with impaired functional status among older people living in a long-term care setting. Geriatr Gerontol Int. 2015;15(3):296-301. https://doi.org/10.1111/ ggi.12272

4. Saga S, Vinsnes AG, Mørkved S, Norton C, Seim A. What characteristics predispose to continence in nursing home residents? A population-based cross-sectional study. Neurourol Urodyn. 2015;34(4):362-7. https://doi. org/10.1002/nau.22563

5. Morsch P, Pereira GN, NavarroJHN, Trevisan MD, Lopes DGC, Bós AJG. Clinical characteristics and social determinants in a sample of non-homebound elderly cad. Saúde Publica. 2015;31(5):1025-34. https://doi.org/10.1590/01021$\underline{311 \times 00053014}$

6. Kwon CS, Lee JH. Prevalence, risk factors, quality of life, and health-care seeking behaviors of female urinary incontinence: results from the 4th Korean National Health and Nutrition Examination Survey VI (2007-2009). Int Neurourol J. 2014;18(1):31-6. https://doi.org/10.5213/ inj.2014.18.1.31

7. Coyne KS, Wein A, Nicholson S, Kvasz M, Chen Cl, Milsom I. Economic burden of urgency urinary incontinence in the United States: a systematic review. J Manag Care Pharm. 2014;20(2):130-40. https://doi.org/10.18553/ jmcp.2014.20.2.130

8. Hrisanfow E, Hägglund D. Impact of cough and urinary incontinence on quality of life in women and men with chronic obstructive pulmonary disease. J Clin Nurs. 2013;22(1-2):97105. https://doi.org/10.1111/j.1365-2702.2012.04143.x

9. Aniuliene R, Aniulis P, Steibliene V. Risk factors and types of urinary incontinence among middle-aged and older male and female primary care patients in Kaunas Region of Lithuania: cross sectional study. Urol J. 2016;13(1):2552-61.

10. Park J, Son Hong G-R. Association of functional ability and benign prostatic hyperplasia with urinary incontinence in older Korean men. Int Neurourol J. 2016;20(2):137-42. https://doi.org/10.5213/inj.1630432.216

11. Léon $P$, Chartier-Kastler E, Rouprêt M, Ambrogi V, Mozer $P$, Phé $V$. Long-term functional outcomes after artificial urinary sphincter implantation in men with stress urinary incontinence. BJU Int. 2015;115(6):951-7. https://doi. org/10.1111/bju.12848

12. Haylen BT, de Ridder D, Freeman RM, Swift SE, Berghmans B, Lee J, et al. An International Urogynecological Association (IUGA)/International Continence Society (ICS) joint report on the terminology for female pelvic floor dysfunction. Neurourol Urodyn. 2010;29:4-20. https://doi. org/10.1002/nau.20798
13. Jung HB, Kim HJ, Cho ST. A current perspective on geriatric lower urinary tract dysfunction. Korean J Urol. 015;56(4):26675. https://doi.org/10.4111/kju.2015.56.4.266

14. Jerez-Roig J, Souza DLB, Lima KC. Urinary incontinence in institutionalized elderly in Brazil: an integrative review. Rev Bras Geriatr Gerontol. 2013;16(4):865-79. https://doi. org/10.1590/S1809-98232013000400020

15. Mandl M, Halfens RJ, Lohrmann C. Incontinence care in nursing homes: a cross-sectional study. J Adv Nurs. 2015;71(9):2142-52. https://doi.org/10.1111/jan.12676

16. Bertolucci PHF, Brucki SMD, Campacci SR, Juliano Y. The Mini-Mental State Examination in an outpatient population: influence of literacy. Arq de Neuro-Psiquiatr. 1994;52(1):1-7. https://doi.org/10.1590/50004-282X1994000100001

17. Herr M, Grondin H, Sanchez S, Armaingaud D, Blochet C, Vial A, et al. Polypharmacy and potentially inappropriate medications: a cross-sectional analysis among 451 nursing homes in France. Eur J Clin Pharmacol. 2017;73(5):601-8. https://doi.org/10.1007/s00228-016-2193-z

18. Ministério da Saúde (BR). Agência Nacional de Vigilância Sanitária (Anvisa). Resolução da Diretoria Colegiada - RDC/ Anvisa n² 283 de 26 de setembro de 2005 [Internet]. Brasília, DF; 2005 [citado 4 Dez 2018]. Disponível em: http://www20. anvisa.gov.br/segurancadopaciente/index.php/legislacao/ item/rdc-283-de-26-de-setembro-de-2005

19. Brucki SMD, Mansur LL, Carthery-Goulart MT, Nitrini R. Formal education, health literacy and Mini-Mental State Examination. Dement Neuropsychol. 2011;5(1):26-30. https://doi.org/10.1590/S1980-57642011DN05010005

20. Ministério da Saúde (BR). Conselho Nacional de Saúde. Comitê Nacional de Ética em Pesquisa em Seres Humanos. Resolução nº 466 de 12 de dezembro de 2012 [Internet]. Brasília, DF; 2012. [citado 4 Dez 2018]. Disponível em: http:// conselho.saude.gov.br/resolucoes/2012/Res0466.pdf

21. Jerez-Roig J, Santos MM, Souza DL, Amaral FL, Lima KC. Prevalence of urinary incontinence and associated factors in nursing home residents. Neurourol Urodyn. 2016;35(1):1027. https://doi.org/10.1002/nau.22675

22. Pitangui $A C R$, Silva RG, Araújo RC. Prevalence and impact of urinary incontinence on the quality of life of institutionalized elderly women. Rev Bras Geriatr Gerontol. 2012;15(4):61926. https://doi.org/10.1590/S1809-98232012000400002

23. Rosa LHT, Souza CM, Lima CHL, Boggio ESB, Santos FC, Carboni C, et al. Prevalência da incontinência urinária em idosos de Porto Alegre-RS. Geriatr Gerontol Aging. 2014;8(2):104-9.

24. Silva VA, D'Elboux MJ. Factors associated with urinary incontinence in elderly individuals who meet frailty criteria. Texto Contexto Enferm. 2012;21(2):338-47. https://doi. org/10.1590/s0104-07072012000200011

25. Wang C, Wan X, Wang K, Li J, Sun T, Guan X. Disease stigma and intentions to seek care for stress urinary incontinence among community-dwelling women. Maturitas. 2014;77(4):351-5. https://doi.org/10.1016/j.maturitas.2014.01.009

26. Hajek A, Brettschneider C, Lange C Posselt T, Wiese $B$, Steinmann $S$, et al. Longitudinal predictors of institutionalization in old age. PLoS One. 2015;10(12):1-11. https://doi.org/10.1371/journal.pone.0144203 
27. Mouodi S, Bijani A, Hosseini SR, Hajian-Tilaki K. Gender differences in the health status of elderly living alone compared to those who are not alone: evidence of the AHAP study, North of Iran. Caspian. J. Intern. Med. 2016;7(2):126-32.

28. Maher RL, Hanlon JT, Hajjar ER. Clinical consequences of polypharmacy in elderly. Expert Opin Drug Saf. 2014;13(1):57-65. https://doi.org/10.1517/14740338.2013. 827660

29. Marques LP, Schneider IJC, Giehl MWC, Antes DL, d'Orsi E. Demographic, health conditions, and lifestyle factors associated with urinary incontinence in elderly from Florianópolis, Santa Catarina, Brazil. Rev Bras Epidemiol. 2015;18(3):595-606.https://doi.org/10.1590/19805497201500030006

30. Griebling TL. Re: Prevalence of commonly prescribed medications potentially contributing to urinary symptoms in a cohort of older patients seeking care for incontinence. J Urol. 2014;192(2):490-1. https://doi.org/10.1016/j. juro.2014.05.083
31. Shaw C, Wagg A. Urinary incontinence in older adults. Medicine. 2017;45(1):23-7. https://doi.org/10.1016/j. mpmed.2016.10.001

32. Schumpf LF, Theill N, Scheiner DA, Fink D, Riese F, Betschart C. Urinary incontinence and its association with functional physical and cognitive health among female nursing home residents in Switzerland. BMC Geriatrics. 2017;17:17. https://doi.org/10.1186/s12877-017-0414-7

33. Cucato GG, Ritti-Dias RM, Cendoroglo MS, Carvalho JMM, Nasri F, Costa MLM, et al. Health-related quality of life in Brazilian community-dwelling and institutionalized elderly: comparison between genders. Rev Assoc Med Bras. 2016;62(9):848-52. https://doi.org/10.1590/18069282.62.09.848

34. Teunissen DT, Stegeman MM, Bor HH, Lagro-Janssen TA. Treatment by a nurse practitioner in primary care improves the severity and impact of urinary incontinence in women. An observational study. BMC Urol. 2015;15:51. https://doi. org/10.1186/s12894-015-0047-0 